\title{
Crossing Intrinsic Depolarizing Resonances in ELSA with Pulsed Betatron Tune Jump Quadrupoles
}

\author{
C. Steier, W. v. Drachenfels, F. Frommberger, M. Hoffmann, D. Husmann, J. Keil, \\ Bonn University, Germany; \\ S. Nakamura, T. Nakanishi, Nagoya University, Japan; H. Sato, T. Toyama, KEK, Japan
}

\section{Abstract}

At the electron stretcher accelerator ELSA of Bonn University an external fixed target experiment using a polarized electron beam has been started. To provide a polarized beam with sufficient intensity a dedicated source for polarized electrons is used (with a GaAs-AlGaAs superlattice photocathode). To prevent depolarization during acceleration in the circular accelerators several depolarizing resonances have to be corrected for. One type of resonances (the intrinsic ones) is compensated using two pulsed betatron tune jump quadrupoles. This method is applied because adiabatic crossing techniques that became popular in proton accelerators in the last years cannot be used in an electron accelerator due to synchrotron radiation effects at higher energies.

\section{INTRODUCTION}

At ELSA [1] external fixed target experiments with longitudinally polarized electrons or circularly polarized photons (produced by Bremsstrahlung) are carried out. For this purpose a polarized electron source is used. With a GaAs superlattice crystal a polarization level of about $66 \%$ at the source has been achieved [2]. The polarized electron beam is preaccelerated in a LINAC and a fast cycling booster $(50 \mathrm{~Hz})$. After injection into the main ring further acceleration up to $3.5 \mathrm{GeV}$ is possible. The polarization of the extracted beam can be determined with a Møller polarimeter.

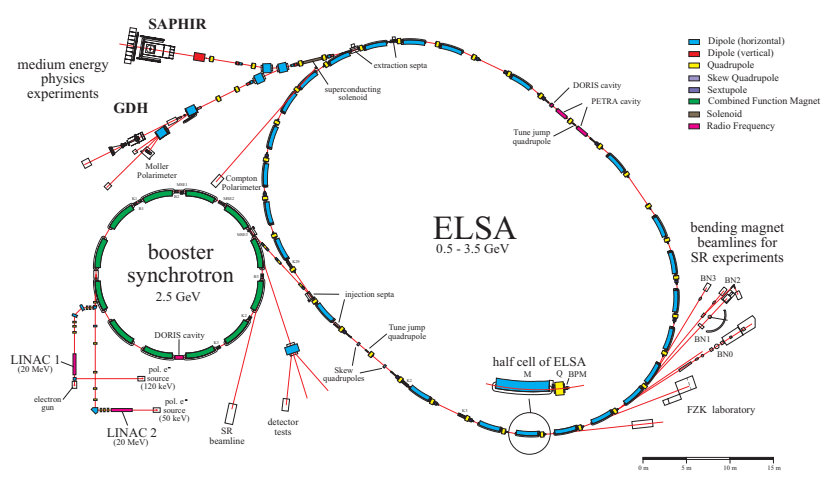

Figure 1: The ELSA facility at Bonn University.

In ELSA depolarization is caused mainly by intrinsic and imperfection resonances [3][4]. The spin originally oriented nearly perpendicular to the accelerator plane precesses around the direction of the magnetic field in the

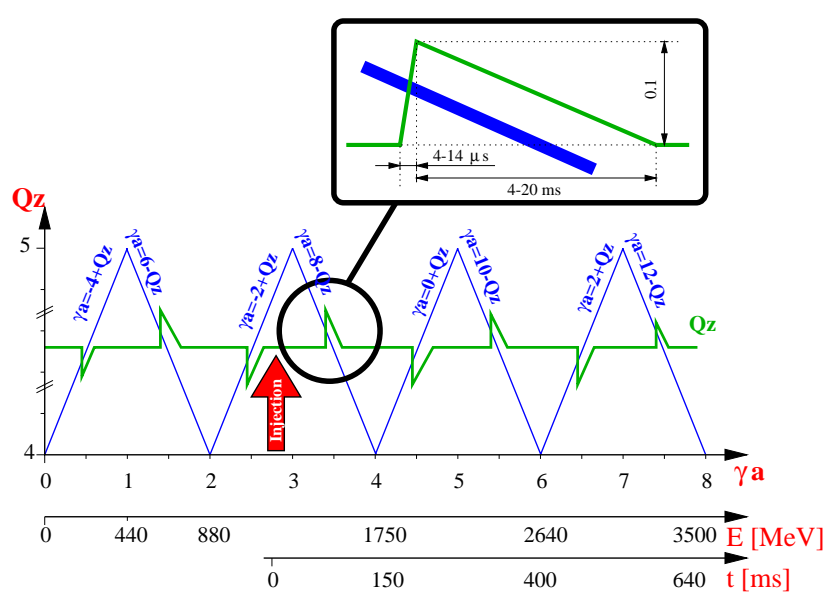

Figure 2: Fast tune jumping of all resonances in ELSA.

bending magnets. During circulation of the particles in the synchrotron only the polarization component parallel to the guiding field is conserved while the other components are lost. So, horizontal magnetic fields may cause polarization loss in the case of a resonance with the spin precession frequency.

Intrinsic resonances are driven by the vertical betatron motion of the electrons, characterized by the vertical betatron tune $Q_{z}$.

Depolarization caused by the linear crossing of an isolated resonance is quantified by the Froissart-StoraFormula [5]

$$
\frac{P_{f}}{P_{i}}=2 e^{-\frac{\pi|\epsilon|^{2}}{2 \alpha}}-1
$$

In this formula the influence of synchrotron radiation is neglected, so it cannot be used for resonances at high energies in electron accelerators. Spin tracking studies show especially that a total spin flip $\left(\frac{P_{f}}{P_{i}}=-1\right)$ is impossible there.

Small polarization losses can be obtained for a small resonance strength $\epsilon$ or a high crossing speed $\alpha=\frac{\dot{\gamma} a \mp \dot{Q}_{z}}{\omega_{0}}$ which can be achieved by fast shifting the vertical betatron tune $Q_{z}$ for intrinsic resonances using pulsed quadrupoles.

For the resonances in ELSA an absolute tune shift of $\Delta Q_{z} \approx 0.1$ is needed with a rise time of about $10 \mu \mathrm{s}$. This tune jumping is illustrated in Fig. 2. Before crossing the resonance, the vertical betatron tune is shifted very fast by the tune jump quadrupoles. 

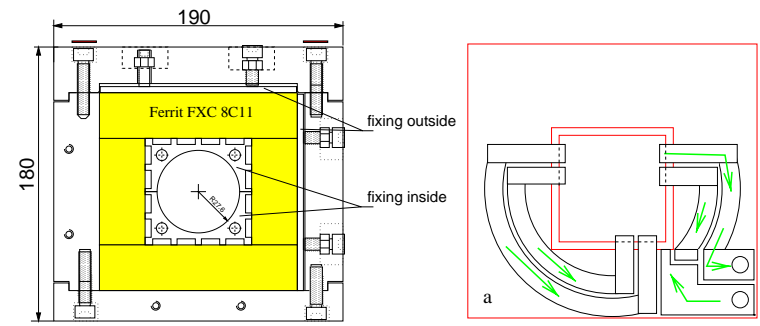

ceramic vacuum chamber
with $10 \mu \mathrm{m}$ titanium coating
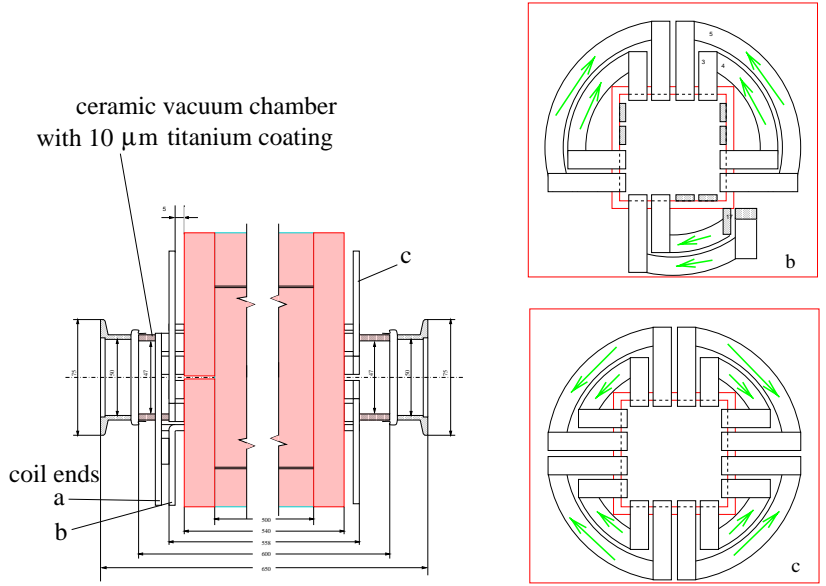

Figure 3: Design of the tune jump quadrupoles: the end cap design consisting of three layers (right), sectional and side view of the magnets (left).

\section{TUNE JUMP MAGNETS}

For ELSA a fast tune jump system was designed. It consists of two quadrupoles which can be pulsed up to $500 \mathrm{~A}$ in 4-14 $\mu \mathrm{s}$. These quadrupoles are of Panofsky type with square shaped ferrite yokes (see Fig. 3) [6]. Calculations for optimization of the design to achieve a low inductance have been applied [7]. Therefore a special end cap design (a,b and c) was carried out. The vacuum chamber is made of $\mathrm{Al}_{2} \mathrm{O}_{3}$ ceramics with a $10 \mu \mathrm{m}$ thin titanium coating on the inner surface. This coating shields the electromagnetic fields of the beam but does not distort the pulsed magnetic field.

The main parameters of the quadrupoles are:

$$
\begin{array}{ll}
\text { resistance: } & (4.298 \pm 0.001) \mathrm{m} \Omega(=) \\
\text { inductance: } & (9.0 \pm 0.1) \mu \mathrm{H}(=) \\
\text { max. pulse current: } & 500 \mathrm{~A} \\
\text { max. field gradient: } & (1.1241 \pm 0.005) \mathrm{T} / \mathrm{m} .
\end{array}
$$

Because of the high resistance of the ferrites there are no significant eddy currents inside the yoke. Frequency dependencies of the impedance lead to a slight dispersion of the pulse shape which has to be compensated by the power supply.

\section{The Power Supplies}

The pulsed power supplies (one for each magnet) were constructed by PPT ${ }^{1}$. A schematic drawing is shown in Fig. 4.

The design concept is similar to the pulsed power supplies used for COSY in Jülich [8].

Each supply consists of three main components, which could be realized in semiconductor technology. First a $1 \mathrm{kV}$ high voltage power supply charges a capacitor bank. The charging is controlled by a PLC (programmable logic controller). An insulated gate bipolar transistor (IGBT) connects the capacitor bank with the load. The IGBT is controlled by the PLC to generate a linear rising edge, which can be varied from 4 to $14 \mu \mathrm{s}$.

The second part consists of a $50 \mathrm{~V}$ power supply and generates the falling edge. It is connected by a MOSFET switch to the load. The falling edge can be varied between 4 and $20 \mathrm{~ms}$.

The third part is a H-bridge, necessary for polarity changes.

The PLC is connected by a VME computer to the control system of the accelerator.

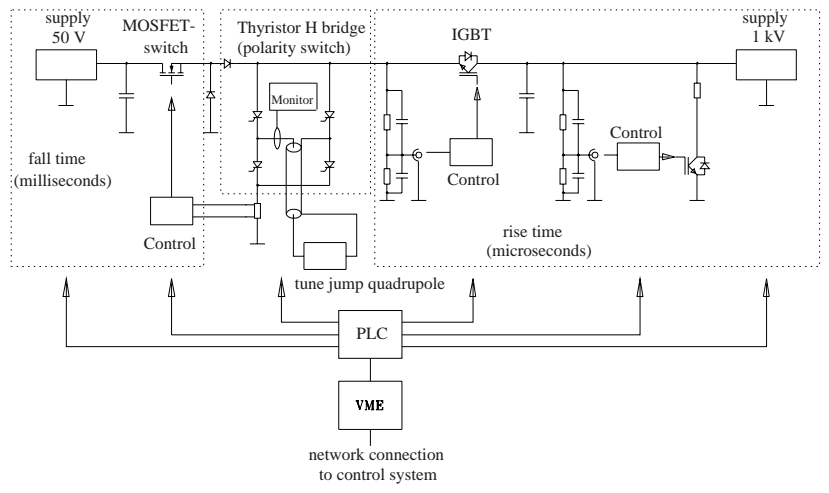

Figure 4: Schematic drawing of the pulsed power supplies.

\section{CALIBRATION AND MEASUREMENTS}

Using a stored beam in ELSA the pulse amplitude of the tune jump magnets was calibrated by jumping onto a half integer betatron resonance $\left(\mathrm{Q}_{z}=4.5\right)$ from various distances with various pulse heights. The betatron tune was measured by analyzing the spectrum of the vertical beam oscillations. When jumping onto the resonance significant beam loss was observed, reducing the lifetime of the beam from $4 \mathrm{~h}$ to less than $10 \mathrm{~min}$.

The measured value of $\frac{\Delta Q_{z}}{\Delta I}=(1.77 \pm 0.08) \cdot 10^{-4} \mathrm{~A}^{-1}$ at $2.3 \mathrm{GeV}$ is in good agreement with the calculations.

Calculations and measurements concerning closed orbit distortions and emittance growth due to the change of the optical functions were carried out [9].

\footnotetext{
${ }^{1}$ Puls Plasma Technik, Dortmund, Germany
} 


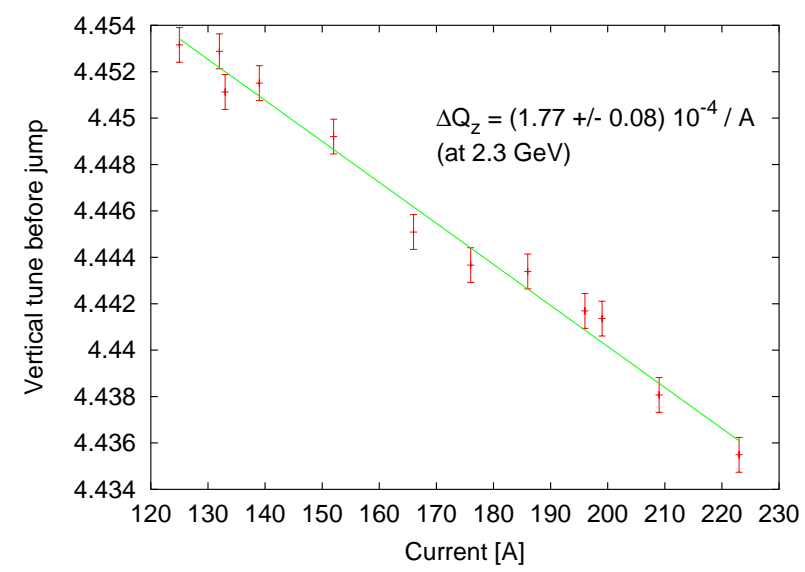

Figure 5: Calibration of the pulsed quadrupoles using a jump onto a half integer betatron resonance.

Fig. 6 shows the maximum distortion of the closed orbit caused by a tune jump of $\Delta Q_{z}=0.1$ at $1.5 \mathrm{GeV}$ during the energy ramp. The rise time of the pulse was $10 \mu$ s and the fall time $20 \mathrm{~ms}$.

The distortions are all smaller than $1 \mathrm{~mm}$ and can therefore be neglected.
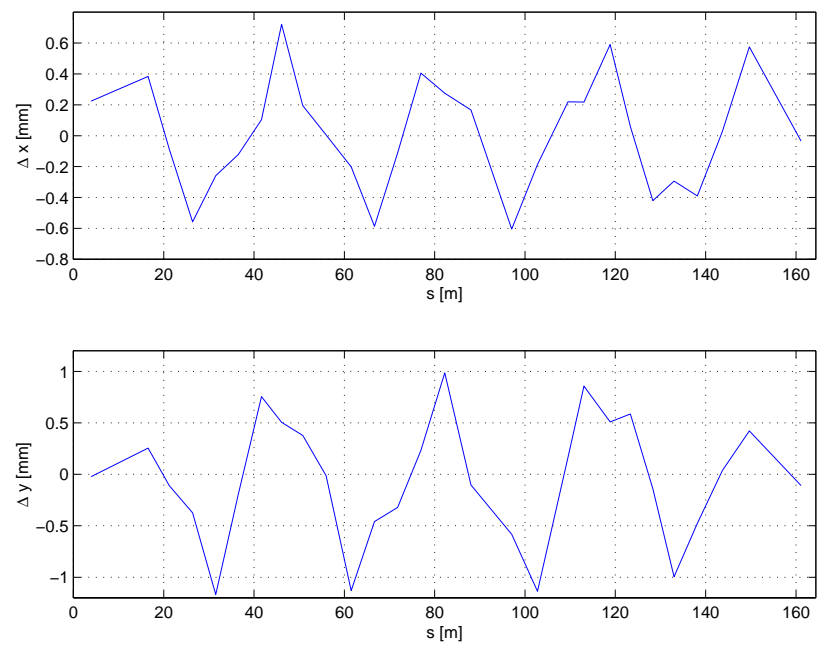

Figure 6: Change of the horizontal and vertical closed orbit during a tune jump for the uncorrected closed orbit.

The successful operation of the tune jump magnets was tested first on the intrinsic resonance at $2.0 \mathrm{GeV}$ [9]. Without tune jump the resonance causes a depolarization of $25 \%$. Using the pulsed quadrupoles the depolarization could be minimized to less than $2 \%$. Variation of the trigger time of the tune jump magnets leads to the curve shown in Fig. 7. If the jump is triggered too early stronger depolarization could be observed, which is expected because the resonance crossing speed is lower on the falling edge of the pulse than without any pulse. The width of the optimal time window changes with the pulse amplitude. A higher pulse amplitude broadens the window and therefore minimizes sensitivity to time shifts. However, too large amplitudes, may cause beam loss, because of jumping onto a neighbouring betatron resonance.

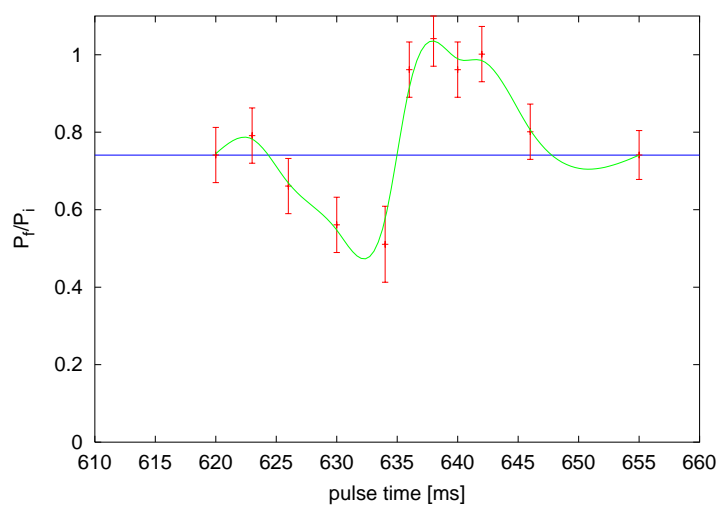

Figure 7: Jumping over the $\left(0+Q_{z}\right)$-resonance: Polarization dependency of the jump time. The pulse amplitude was $-110 \mathrm{~A}$ with each magnet, corresponding to a $\Delta Q_{z}=-0.047$.

\section{SUMMARY}

Polarized electrons have been accelerated at ELSA without significant losses in polarization degree. In the case of crossing intrinsic resonances a fast betatron tune jump magnet system has provided the conservation of polarization. First measurements have shown that the depolarization due to the strong intrinsic resonance at $2.0 \mathrm{GeV}$ can be reduced to less than $2 \%$ with the tune jump magnets.

Further investigations will follow soon to adjust the amplitudes and trigger times to minimize the influence of the remaining intrinsic resonances. Together with a harmonic correction of the imperfection resonances in ELSA a polarized beam will be provided up to the maximum energy in near future.

\section{REFERENCES}

[1] K.H. Althoff et al., Part. Acc. 27 (1990) 101

[2] S. Nakamura et al., AIP Conf. Proc. 421, Urbana (1997) 497

[3] C. Steier, D. Husmann, Proc. 1997 Part. Accel. Conf., Vancouver

[4] S. Nakamura et al., Nucl. Instr. \& Meth. A 441 (1998) 93

[5] M. Froissart and R. Stora, Nucl. Instr. \& Meth. 7 (1960) 297

[6] M. Hoffmann, diploma thesis, BONN-IB-98-10

[7] T. Toyama, C. Steier et al., BONN-ME-97-01

[8] A. Lehrach, PhD thesis, Jülich, IKP-98-12-034 (1997)

[9] C. Steier, PhD thesis, BONN-IR-99-07 (1999) 